\title{
因HAD
}

DOI: http://doi.org/10.22585/hospdomic.v3i1.62

\section{Desarrollo de la gestión del proceso general de la manipulación de los medicamentos peligrosos en las unidades de hospitalización a domicilio}

\section{Development of the management of the general process of the handling of hazardous drugs in the home hospitalization units}

Maria A. Bernabeu Martinez',2, Pedro García Salom³, Amparo Burgos San José3, Andrés Navarro Ruiz ${ }^{4}$, Javier Sanz Valero ${ }^{1,6}$, Carmina Wanden-Berghe ${ }^{5,6}$

1. Universidad Miguel Hernández. Departamento de Salud Pública e Historia de la Ciencia, Campus de Sant Joan d'Alacant. Alicante. España.

2. Hospital General Universitario de Alicante. Servicio de Farmacia de Área. Alicante. España.

3. Hospital General Universitario de Alicante. Servicio de Farmacia. Alicante. España.

4. Hospital General Universitario de Elche. Servicio de Farmacia. Alicante. España.

5. Hospital General Universitario de Alicante. Unidad De Hospitalización a Domicilio. Alicante. España.

6. Instituto de Investigación Sanitaria y Biomédica de Alicante (ISABIAL-FISABIO). Alicante. España.

Correspondencia/Correspondence

Carmina Wanden-Berghe

carminaw@telefónica.net

Recibido/Received

28.11 .2018

Aceptado/Accepted

02.01 .2019
Conflicto de Intereses/Competing interest Las autoras y autores de este trabajo declaran la inexistencia de conflicto de interés en el presente estudio.

\begin{abstract}
Agradecimientos/Acknowledgments
Este trabajo cuenta con una ayuda del Instituto de Salud Carlos III de Madrid, España, mediante el

Proyecto de Investigación en Salud con referencia PI16/00788.
\end{abstract}

CÓMO CITAR ESTE TRABAJO | HOW TO CITE THIS PAPER

Bernabeu Martinez MA, García Salom P, Burgos San José A, Navarro Ruiz A, Sanz Valero J, WandenBerghe C. Desarrollo de la gestión del proceso general de la manipulación de los medicamentos peligrosos en las unidades de hospitalización a domicilio. Hosp Domic. 2019;311):9-23. 


\section{RESUMEN}

Objetivo: Identificar los peligros asociados y describir los riesgos químicos derivados de éstos, en el proceso de manipulación de los medicamentos peligrosos (MP) en las Unidades de Hospitalización a Domicilio (UHD), como fase inicial de su evaluación de riesgos y que afectan a la seguridad del profesional sanitario.

Método: Se realizó un consenso de expertos (grupo nominal y técnicas documentales) utilizando un método mixto mediante dos rondas presenciales (reunión de los participantes y aprobación de propuestas) y tres rondas enmascaradas (revisión del material de forma individual). El análisis se aplicó al ámbito de la Hospitalización a Domicilio y las etapas del proceso se diseñaron mediante notación gráfica normalizada Business Process Modeling Notation.

Resultados: Se obtuvo el diagrama de flujo específico para la gestión y trazabilidad de los MP, caracterizándose cada una de las fases del proceso general, recopiladas en un cuadro de gestión de etapas y operaciones de conservación, transporte y administración de MP en las unidades de hospitalización a domicilio, que sirvió para la posterior descripción de riesgos químicos y vías de exposición.

Conclusiones: Los MP deben integrarse en un sistema normalizado de gestión con el fin de mejorar la seguridad del paciente y de los profesionales sanitarios, a la vez que se maximiza la eficiencia de los recursos y minimizan los incidentes procesales, garantizando la calidad y la seguridad del proceso de manipulación de MP en la UHD.

Sería deseable, una vez se han identificado los peligros, llevar a cabo una evaluación de los riesgos siguiendo una metodología sistemática y de abordaje preventivo que permita calibrar la probabilidad de ocurrencia y la gravedad de cualquier suceso adverso.

Palabras clave: Servicios de Atención a Domicilio Provisto por Hospital; Sustancias Peligrosas; Antineoplásicos; Citostáticos; Salud laboral; Control de calidad; Evaluación del proceso; Gestión de la información; Exposición Ocupacional.

\section{ABSTRACT}

Objective: To identify associated hazards and to describe chemical risks arising from these in the process of handling of hazardous drugs (HD) in Home Hospitalization Units (HHU), as the initial phase of its risk assessment and which affect the security of healthcare professionals.

Method: There was a consensus of experts (nominal group and documentary techniques) using a mixed method through two face-to-face rounds (meeting of participants and approval of proposals) and three masked rounds (review of the material on an individual basis). The analysis was applied to the field of home hospitalization and the stages of the process were designed using standardized graphical notation Business Process Modeling Notation.

Results: It was obtained the specific flowchart for management and traceability of the HD, being characterized each of the phases of the general process, they were collected in a chart of stage management and operations of conservation, transportation and administration of HD in $\mathrm{HHU}$, which served for the subsequent description of chemical hazards and exposure ways.

Conclusions: The HD should be integrated in a standard management system in order to improve the safety of the patient and healthcare professionals, at the same time that the efficiency of resources are maximized and procedural incidents are minimized, ensuring the quality and the safety of the process of handling the HD on the HHU.

It would be desirable, once the hazards have been identified, to carry out an assessment of the risks by following a systematic methodology and preventative approach that allows calibrating the probability of occurrence and severity of any adverse event.

Keywords: Home Care Services, HospitalBased; Hazardous Substances; Antineoplastic Agents; Cytostatic Agents; Occupational Health; Quality control; Process assessment; Information management; Occupational Exposure. 


\section{INTRODUCCIÓN}

El sector sanitario constituye uno de los sectores más expuestos a los riesgos laborales, tal y como establece la Agencia Europea para la Seguridad y Salud en el Trabajo (EU-OSHA) (1). Quizá, sean los riesgos radiológicos y biológicos los peligros más conocidos entre los profesionales de la salud, aunque no exclusivos y, por ello, tanto las medidas de seguridad como la sensibilización ante este tipo de eventualidades son relativamente altas. Sin embargo, no hay que olvidar el riesgo químico, tanto aquel que supone un riesgo inmediato -lo que en inglés se describe como «dangerous»- como el relacionado con el daño a largo plazo, a exposiciones sucesivas y acumuladas -lo que en inglés se conoce como «hazardous»- (2). Es en este segundo escenario donde se enmarcan los medicamentos peligrosos (MP), descritos como el mayor peligro químico presente en el sector sanitario, siendo los productos citotóxicos uno de los agentes químicos más peligrosos jamás desarrollados (3).

El término "Medicamento Peligroso» es una traducción aproximada del original en inglés "Hazardous Drug» y fue utilizado por primera vez en los años 90 por la American Society of Hospital Pharmacists (ASHP) (4). Este término englobaba tanto los fármacos citotóxicos, para los que ya existía concienciación de su peligrosidad por parte de los sanitarios, como aquellos fármacos que, no utilizándose en terapia anticancerosa, podían provocar efectos similares sobre los profesionales expuestos. Posteriormente, en 2004, el National Institute for Occupational Safety and Health (NIOSH) adoptó esta terminología, considerando MP cualquier fármaco que hubiera demostrado: efecto carcinogénico, teratogénico, genotóxico, toxicidad reproductiva o para el desarrollo o en órganos a dosis bajas, tanto en estudios en animales, como en humanos (5).

En consecuencia, resulta evidente que el proceso de manipulación de los MP, constituido por circuitos de elevada complejidad y con gran número de actores implicados, conlleva importantes riesgos, tanto para el paciente como para el profesional sanitario, resultando imprescindible garantizar la seguridad del proceso. Más aún en el entorno de la atención domiciliaria, donde la precaución, por parte del enfermo y sus cuidadores, puede relajarse y en el cual las condiciones en el lugar de trabajo (la casa del paciente) suelen ser no controladas y carentes, en la mayoría de ocasiones, de supervisión directa (1).

En este sentido, la evaluación de riesgos se torna uno de los puntos clave en la gestión y control del proceso de los MP. Por tanto, es imprescindible determinar los riesgos asociados al proceso, obteniendo la información necesaria para adoptar medidas preventivas. Con este fin, existen múltiples modelos de evaluación de riesgos descritos en la literatura científica (cualitativos, semicuantitativos, simplificados, complejos, etc.) (6), y aunque cada uno presenta sus peculiaridades, todos ellos tienen como común denominador el primer paso, indispensable para su correcta gestión: la identificación de los peligros asociados al proceso.

Por todo lo mostrado, el objetivo de este trabajo fue identificar los peligros asociados y describir los riesgos químicos derivados de éstos, en el proceso de manipulación de los MP en las Unidades de Hospitalización a Domicilio (UHD), como fase inicial de su evaluación de riesgos, dejando constancia de que este trabajo se centra exclusivamente en los peligros que afectan a la seguridad del profesional sanitario. No considera por tanto, los riesgos que puedan afectar al paciente ni a la seguridad ambiental. 


\section{MATERIAL Y MÉTODO}

\section{Diseño}

Se realizó un consenso de expertos (grupo nominal y técnicas documentales) utilizando un método mixto mediante dos rondas presenciales (reunión de los participantes y aprobación de propuestas) y tres rondas enmascaradas (revisión del material de forma individual). El consenso estuvo conformado por un equipo multidisciplinar (4 farmacéuticos/as hospitalarios/as, una médico de UHD y un farmacéutico auditor de salud pública), quienes por su formación académica y experiencia profesional cumplían el criterio de elección establecido: ejercicio profesional, por más de 5 años, en puesto donde se manipulen MP o se realice evaluación de riesgos.

\section{Procedimiento}

El consenso se desarrolló en 7 fases y se detalla a continuación:

- Fase 1: Revisión bibliográfica de los antecedentes y protocolos relacionados con la vigilancia de peligros (retroalimentación controlada), a través de la identificación, recogida y análisis de documentos relacionados con el hecho o contexto estudiado.

- Fase 2: Preparación de la documentación inicial y generación del primer flujograma y su cuadro de gestión.

- Fase 3 (Enmascarada): Revisión de este material y aporte de correcciones.

- Fase 4 (Presencial): Puesta en común de las aportaciones realizadas y corrección de los documentos.

- Fase 5 (Enmascarada): Nueva revisión de los documentos y nuevas aportaciones si se consideraba necesario.

- Fase 6 (Presencial): Aceptación de las últimas revisiones realizadas y generación del material corregido.

- Fase 7 (Enmascarada): Aprobación final del material por unanimidad: flujograma y cuadro de gestión.

Ámbito de aplicación:

- El análisis se aplicó al ámbito de la Hospitalización a Domicilio.

\section{Etapas del proceso}

Se diseñó mediante notación gráfica normalizada Business Process Modeling Notation el flujograma que simboliza la gestión operativa de la cadena logística del MP. Este modelo de gestión, implementado anteriormente por Bernabeu Soria et al. (7), permitió analizar y caracterizar cada uno de los pasos dentro del proceso, lo que facilitó el análisis de cada uno de ellos y la determinación de los posibles peligros. Además, esta técnica fue implantada y verificada con éxito por Cervera Peris et al. (8). Esta metodología permitió que los procesos pudieran ser fácilmente escalados (ampliados), si en algún momento hubiera sido necesario, permitiendo eficiencia y eficacia ante cualquier cambio o nuevo requerimiento. Las etapas a controlar se obtuvieron a partir de la revisión sistemática llevada a cabo por Bernabeu et al. (9): conservación, transporte y administración. 


\section{Comprobación in situ}

A partir de los documentos desarrollados se llevó a cabo la identificación de cada una de las etapas y de sus posibles puntos de control. Posteriormente se verificó en el lugar donde se realizan las operaciones, la correspondencia entre los documentos desarrollados (diagrama de flujo y cuadro de gestión) y las etapas que configuran el proceso de manipulación de MP.

\section{Nomenclatura}

El léxico aceptado y utilizado en la generación de los documentos fue:

- Proceso: conjunto de actividades interrelacionadas que se llevan a cabo de forma ordenada por parte de un grupo de actores para alcanzar un fin predefinido.

- Etapa: cada subproceso existente en el flujograma final.

- Operación: cada una de las actividades o pasos que conforman una etapa.

- Peligro: capacidad intrínseca potencial para causar daño a la salud del profesional sanitario (6), clasificándose según su naturaleza en:

- Peligro físico: objetos o fragmentos de éstos que puedan causar lesiones al trabajador.

- Peligro biológico: cualquier tipo de microorganismo procedente de un paciente que, bien por contacto directo o indirecto (a través de tejidos y/o fluidos del paciente), puedan producir una infección en el profesional sanitario.

- Peligro químico: agente químico (MP) que por su capacidad intrínseca tóxica pueda causar daño al personal que lo manipule.

- Riesgo: posibilidad de que un trabajador sufra un determinado daño derivado de la exposición a un peligro (6), categorizándose en:

- Riesgo físico: cortes con vidrio y otros materiales, pinchazos con objetos punzantes, etc.

- Riesgo biológico: exposición a agentes infecciosos vía parenteral (pinchazos tras administración de un MP a un paciente infectado).

- Riesgo químico: exposición al MP por vía inhalatoria, a través de piel o mucosas, contacto con ojos, ingestión y parenteral.

\section{RESULTADOS}

El promedio de años de experiencia de los/las expertos/as fue de $22 \pm 3,17$, mediana igual a 25,50, con máximo de 28 y mínimo de 8 años; 4 tenían experiencia en manejo de medicamentos peligrosos y 2 en evaluación de riesgos.

\section{Diagrama de flujo y cuadro de gestión}

A partir del consenso de expertos recogido se llegó a la obtención del Proceso Global (figura 1). En esta figura se describieron todas y cada una de las etapas que integraban el procedimiento de gestión de la trazabilidad del MP a través de la UHD, lo que permitiría su seguimiento y reproducibilidad. 
FIGURA 1. PROCESO GLOBAL: FLUJOGRAMA DE GESTIÓN DE LOS MEDICAMENTOS PELIGROSOS (MP) EN UNIDADES DE HOPITALIZACIÓN DOMICILIARIA (UHD) (PARTE 1/2)

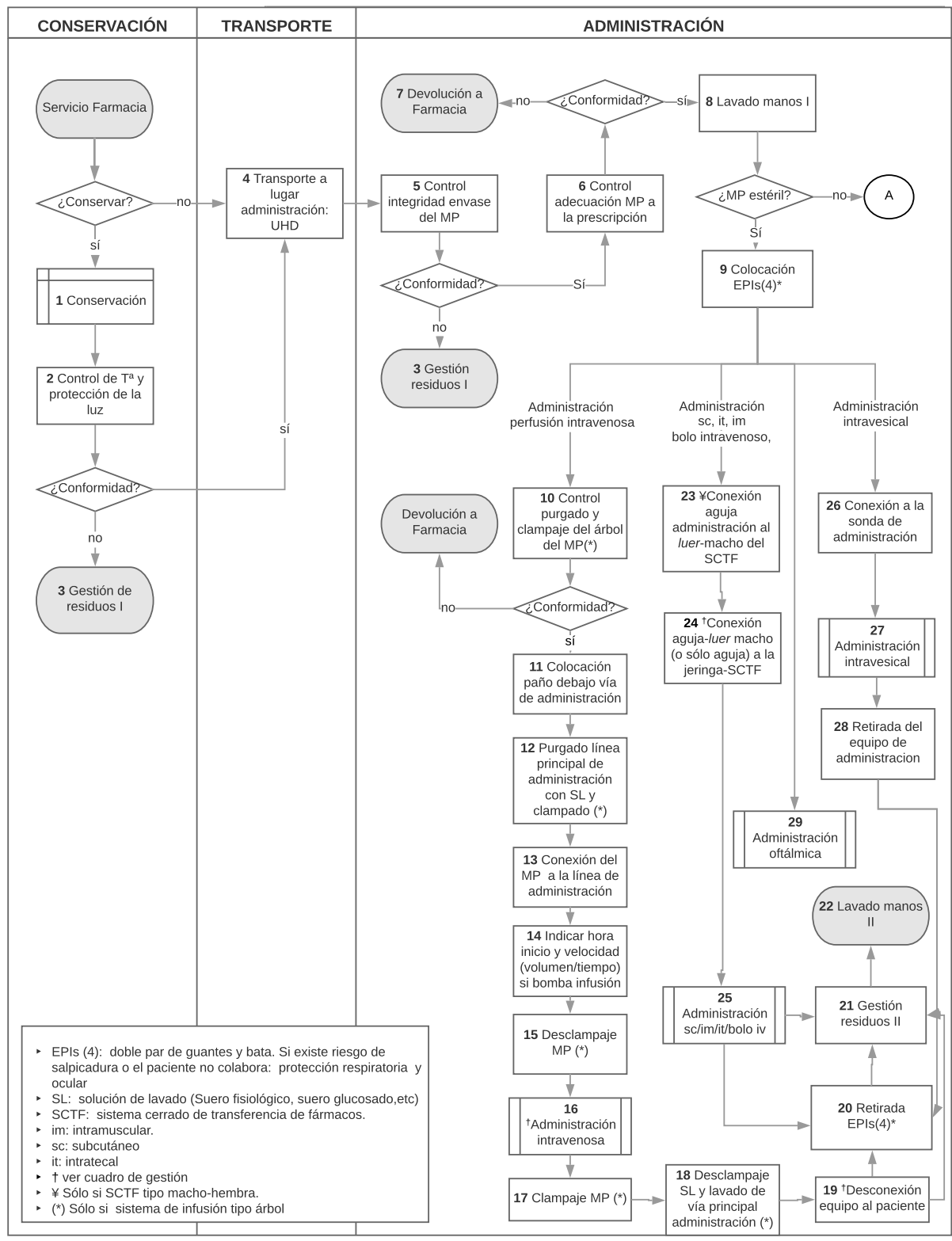


Desarrollo de la gestión del proceso general de la manipulación...

FIGURA 1. PROCESO GLOBAL: FLUJOGRAMA DE GESTIÓN DE LOS MEDICAMENTOS PELIGROSOS (MP) EN UNIDADES DE HOPITALIZACIÓN DOMICILIARIA (UHD) (PARTE 2/2)

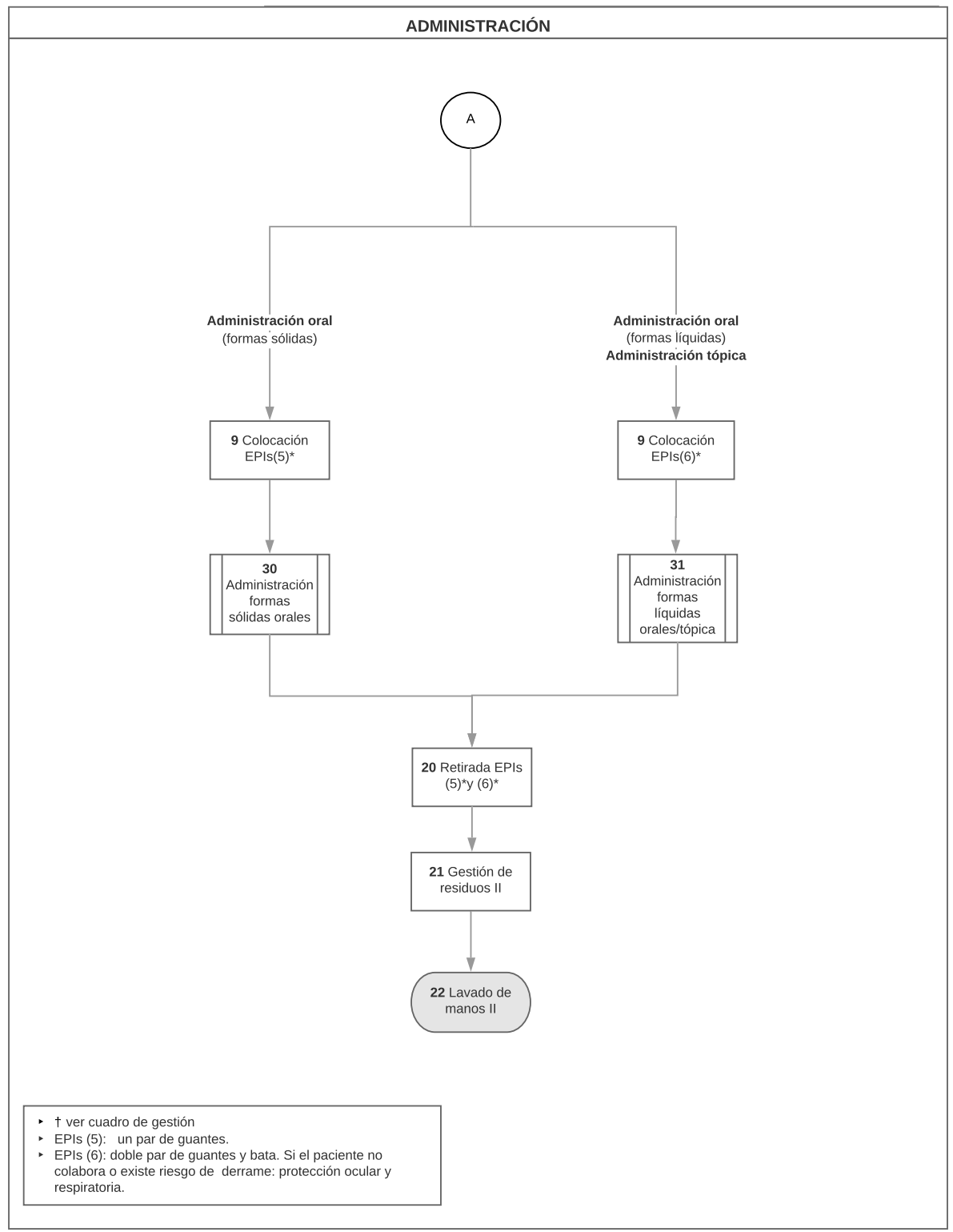


Cada una de las etapas fue recogida en un cuadro de gestión, con el fin de sistematizar y facilitar la comprensión de los resultados, codificándose para cada etapa del proceso las siguientes variables (tabla 1): etapa, operación y número de operación (para facilitar su identificación en los diferentes documentos), lugar en el que se realiza la operación (dónde), persona que desempeña la actividad (quién), presencia de peligro (sí/no) y tipo de peligro identificado (químico, físico, biológico).

Se establecieron 31 operaciones correspondientes a las tres etapas del proceso de manipulación llevadas a cabo en las UHD (3 (0,1\%) operaciones en la conservación; 1 (0,03\%) actividad durante transporte y $27(87,1 \%)$ correspondientes a la etapa de administración).

\section{Determinación de los posibles peligros}

Del estudio del cuadro de gestión, se identificó algún tipo de peligro en 15 operaciones (48,4\%). La distribución de estos peligros según su naturaleza fue: 6 operaciones con peligro físico (19,4\%), 2 operaciones en las que se identificó peligro biológico (0,06\%) y 15 operaciones con peligro químico (48,4\%); ver tabla 1.

A partir del cuadro de gestión general, se seleccionaron aquellas operaciones en las que se identificó algún peligro químico, elaborándose una tabla en la que se describieron los riesgos derivados de éstos y las posibles vías de exposición al MP (tabla 2).

\section{DISCUSIÓN}

El desarrollo del flujograma del Proceso Global (cuadro de gestión), como elemento de representación visual de la gestión operativa de la cadena logística del MP en la UHD, permitió reconocer, en primer lugar, la extraordinaria complejidad del circuito de manipulación de MP en las unidades clínicas de atención al paciente y, en segundo lugar, la multidisciplinaridad de este proceso. Este tipo de representación resultó fundamental para el conocimiento de las distintas etapas, pues condensa mucha información en poco espacio y facilita una rápida y eficaz comprensión y comprobación de las actividades que conforman el proceso, así como el orden en qué deben realizarse.

Atendiendo a la distribución de las operaciones en las tres etapas llevadas a cabo en la UHD, llamó la atención que un gran número de operaciones totales correspondían a la etapa de administración. Este hecho no es de extrañar, pues a la multiplicidad de vías de administración hay que añadir que la administración de MP, junto con la preparación y la gestión de residuos, son las fases del proceso de manipulación de MP consideradas por NIOSH como de mayor riesgo laboral (5). Esto explica la existencia de múltiples operaciones de control y protección (operaciones 5, 6 y 8 a 11), así como la utilización de dispositivos y equipos sofisticados que permiten reducir al mínimo posible la contaminación del área de trabajo y del medio ambiente, y por ende garantizar la seguridad de los trabajadores.

El riesgo principal e inherente a la utilización de los MP es el químico, debido a las características intrínsecas de peligrosidad que los definen (carcinogenicidad, teratogenicidad, genotoxicidad, toxicidad reproductiva o para el desarrollo o en órganos a dosis bajas). No obstante, el hecho de que durante las etapas de conservación, transporte y administración se manipulen objetos cortantes y punzantes (como envases de vidrio que contienen el MP y agujas para la administración), así como el contacto directo con el enfermo y/o fluidos de éste durante la etapa de administración, determinó la existencia adicional, aunque minoritaria, de peligros físicos (operaciones 3 a 5, 7, 21 y 25) y biológicos (operaciones 21 y 25). 
Por el contrario, si bien es cierto que existe la posibilidad de que los peligros físicos se materialicen (cortes con envases de vidrios o fragmentos de éstos y/o pinchazos con agujas) es importante apuntar que en la práctica, este tipo de riesgos se han minimizado enormemente debido a la expansión de la utilización de dispositivos y equipos sin agujas, conexiones luer-lock y al uso generalizado de envases destinados a contener los MP de materiales plásticos tipo poliolefinas, siendo anecdótica la utilización del vidrio, que queda relegado a situaciones puntuales de problemas de incompatibilidad del MP.

El peligro biológico asociado a la manipulación de MP se produce tras pinchazo accidental con aguja después de la administración de un MP a un paciente infectado. Por tanto, este tipo de riesgo sólo se observa en la etapa de administración, y aún más, únicamente en aquel tipo de administración en el que se requieren agujas (subcutánea, intramuscular, bolo intravenoso o intratecal), y durante la gestión de residuos post-administración.

Por otra parte, es importante destacar que ciertas operaciones pueden presentar distinto riesgo químico en función del tipo de equipo o dispositivo que se utilice, tal y como queda reflejado en el cuadro de gestión, mediante el desdoble de dichas operaciones (operación 16, 19 y 24).

Las consecuencias de estos riesgos quedan evidenciadas en la bibliografía, la cual refleja que la exposición laboral a los MP puede inducir la aparición de efectos adversos agudos o crónicos, como erupciones, trastornos reproductivos o posibles alteraciones cromosómicas (10). Las vías de exposición descritas son la inhalación, absorción a través de piel o mucosas, exposición ocular, ingestión e inyección, produciéndose fundamentalmente tras situaciones de contaminación del envase que contiene el MP o goteo de éste, derrames, salpicaduras, generación de polvos o aerosoles al manipularlos (descripción de riesgos y vía de exposición).

Dada la magnitud del problema, no es de extrañar que la preocupación por los riesgos químicos asociados a la manipulación de los MP sea de índole internacional. Prueba de ello es que distintos organismos gubernamentales y no gubernamentales, sociedades científicas y de expertos hayan establecido recomendaciones para la manipulación segura de los MP e insten a los responsables de las organizaciones sanitarias a la realización de una evaluación de los riesgos asociados al circuito de utilización de los MP (3,11-15). De todos ellos, y salvo omisión, únicamente el grupo de consenso americano para los MP «The Hazardous Drug Consensus Group» establece una propuesta metodológica concreta para llevar a cabo dicho análisis de riesgos (12). Identificar el peligro, en cualquier caso, supone el primer paso para mejorar la seguridad laboral de todos los colectivos que pudieran verse expuestos a este tipo de sustancias en ámbito sanitario.

Por todo lo anteriormente expuesto, se podría concluir que los MP deben integrarse en un sistema normalizado de gestión con el fin de mejorar la seguridad del paciente y de los profesionales sanitarios, a la vez que se maximizan la eficiencia de los recursos y minimizan los incidentes procesales. Con el sistema propuesto, se permite establecer un sistema global cuyas etapas quedan totalmente caracterizadas con el fin de garantizar la calidad y la seguridad del proceso de manipulación de MP en la UHD.

Sería deseable, una vez se han identificado los peligros, llevar a cabo una evaluación de los riesgos siguiendo una metodología sistemática y de abordaje preventivo que permita calibrar la probabilidad de ocurrencia y la gravedad de cualquier suceso adverso. 


\section{BIBLIOGRAFÍA}

1. Jong T de, Pawlowska-Cyprysiak K, Hildt-Ciupińska K, Bos E, Nicolescu G, Trifu A, et al. Current and emerging occupational safety and health (OSH) issues in the healthcare sector, including home and community care: European Risk Observatory Report. Luxembourg: European Agency for Safety and Health at Work; 2015.

2. Herrero Alonso JM, Gaspar Carreño M, Cercós Lleti AC. Medicamentos peligrosos: una carrera de fondo. Rev OFIL. 2018;28(1):9-10.

3. Erce A, editor. Preventing occupational exposure to cytotoxic and other hazardous drugs: European Policy Recommendations. [monografía en Internet]. Brussels, Belgium: Rodhe Public Policy; 2016 [citada 5 nov 2018]. Disponible en: https://bit.ly/2qXKWHG

4. American Society of Hospital Pharmacists. ASHP technical assistance bulletin on handling cytotoxic and hazardous drugs. Am J Hosp Pharm. 1990;47(5):1033-49. PMID: 2186621

5. Burroughs GE, Connor TH, McDiarmid MA, Mead KR, Power LA, Reed LD. NIOSH Alert: preventing occupational exposure to antineoplastic and other hazardous drugs in health care settings. Atlanta, USA: National Institute of Occupational Safety and Health (NIOSH), Department of Health and Human Services, Center for Disease Control and Prevention; 2004. Report No.: 2004-165.

6. Instituto Nacional de Seguridad e Higiene en el Trabajo (INSHT). Guía técnica para la evaluación y prevención de los riesgos relacionados con los agentes químicos presentes en los lugares de trabajo. Madrid, España: INSHT; 2013.

7. Bernabeu Soria B, Mateo García M, Wanden-Berghe C, Cervera Peris M, Piñeiro Corrales G, Sanz-Valero J. Development of the management for parenteral nutrition traceability in a standard hospital. Farm Hosp. 2015;39(6):358-68. DOI: 10.7399/fh.2015.39.6.9689; PMID: 26618380

8. Cervera Peris M, Alonso Roris VM, Santos Gago JM, Álvarez Sabucedo LM, Wanden-Berghe C, Sanz Valero J. Implantación de un sistema mSalud para la gestión y trazabilidad de la nutrición parenteral domiciliaria. Hosp Domic. 2017 Jul 28;1(3):129-39. DOI: 10.22585/hospdomic. v113.19

9. Bernabeu-Martínez MA, Ramos Merino M, Santos Gago JM, Álvarez Sabucedo LM, WandenBerghe C, Sanz-Valero J. Guidelines for safe handling of hazardous drugs: A systematic review. PloS One. 2018;13(5):e0197172. DOI: 10.1371/journal.pone.0197172; PMID: 29750798

10. Poveda JL, coordinador. Monografías de farmacia hospitalaria y atención primaria: Medicamentos peligrosos. Barcelona, España: Bayer Hispania SL; 2016.

11. International Society of Oncology Pharmacy Practicioners Standards Committee. ISOPP Standards of Practice: Safe Handling of Cytotoxics. J Oncol Pharm Pract. 2007;13 Suppl:1-81. DOI: 10.1177/1078155207082350; PMID: 17933809

12. The Hazardous Drug Consensus Group. Consensus Statement on the Handling of Hazardous Drugs: Per USP Chapter [monografía en Internet]. North Carolina, USA: Hazardous Drug Consensus Statement; 2017 [citada 5 nov 2018]. Disponible en: https://bit.ly/2qubr6V

13. Poveda JL, Zamudio A, Cobos JL, Muedra M, Aparicio J, Barragán MB, et al. Documento de Consenso de Sociedades Científicas: Seguridad del paciente y del profesional sanitario en la preparación y administración de medicinas peligrosas. Madrid, España: Sociedades Científicas Españolas; 2015. 
14. Real Decreto 665/1997, de 12 de mayo, sobre la protección de los trabajadores contra los riesgos relacionados con la exposición a agentes cancerígenos durante el trabajo. Boletín Oficial del Estado, no 124, (24 de mayo de 1997).

15. Real Decreto 374/2001, de 6 de abril, sobre la protección de la salud y seguridad de los trabajadores contra los riesgos relacionados con los agentes químicos durante el trabajo. Boletín Oficial del Estado, no 104, (1 de mayo de 2001). 


\begin{tabular}{|c|c|c|c|c|c|c|c|c|}
\hline Etapas & Núm. & Operaciones & Dónde & Quién & Peligro & Fisico & Químico & Biológico \\
\hline \multirow[t]{3}{*}{$\begin{array}{l}\text { Conservación } \\
\text { en UHD }\end{array}$} & 1 & $\begin{array}{l}\text { Conservación } \\
\text { (si procede) }\end{array}$ & \multirow{2}{*}{$\begin{array}{l}\text { Neveraffuera de } \\
\text { nevera, separado } \\
\text { del resto de } \\
\text { medicamentos }\end{array}$} & \multirow[t]{3}{*}{ Enfermería } & si & No & SI (pérdida integridad envase) & No \\
\hline & 2 & $\begin{array}{l}\text { Control de temperatura, humedad y condiciones de protección de } \\
\text { la luz }\end{array}$ & & & No & - & - & - \\
\hline & 3 & Gestión residuos I & & & si & $\begin{array}{l}\text { S1 (cortes si envases } \\
\text { de vidrio) }\end{array}$ & SI (exposición si pérdida de integridad de envase, derrame o salpicadura) & No \\
\hline Transporte & 4 & Transporte al lugar de administración & Extra hospitalario & Personal UHD & si & $\begin{array}{l}\text { SI (rotura de recipiente } \\
\text { hermético que contiene } \\
\text { MP) }\end{array}$ & SI (exposición si derrames o pérdida integridad del envase) & No \\
\hline \multirow[t]{5}{*}{$\begin{array}{l}\text { Administración } \\
\text { (etapas comunes } \\
\text { previas) }\end{array}$} & 5 & Control de la integridad del MP & \multirow[t]{43}{*}{ Casa del paciente } & \multirow[t]{43}{*}{ Enfermería } & si & $\begin{array}{l}\text { SI (cortes si envase } \\
\text { vidrio) }\end{array}$ & Sí (exposición si derrames o pérdida de integridad del envase) & No \\
\hline & 6 & Control adecuación del MP a administrar con la prescripción médica & & & NO & NO & No & NO \\
\hline & 7 & Devolución a Farmacia & & & si & $\begin{array}{l}\text { SI (rotura de recipiente } \\
\text { hermético que contiene } \\
\text { MP) }\end{array}$ & Sí (exposición si derrames o pérdida integridad del envase) & No \\
\hline & 8 & Lavado manos 1 & & & No & No & No & No \\
\hline & 9 & $\begin{array}{l}\text { Colocación de EPI (4), (5) ó (6) en función del MP a administrar (ver } \\
\text { flujograma: figura 1) }\end{array}$ & & & No & No & No & NO \\
\hline \multirow{17}{*}{$\begin{array}{l}\text { Administración } \\
\text { perfusión } \\
\text { intravenosa (IV) }\end{array}$} & 10 & $\begin{array}{l}\text { Control purgado y clampaje de la alargadera (o linea secundaria) del } \\
\text { MP (solo si equipo de infusión tipo arbol) }\end{array}$ & & & No & No & No & No \\
\hline & 11 & $\begin{array}{l}\text { Colocación paño } \\
\text { debajo via dei } \\
\text { administración }\end{array}$ & & & No & No & No & No \\
\hline & 12 & $\begin{array}{l}\text { Purgado de la línea principal de } \\
\text { administración } \\
\text { con SL y clampado }\end{array}$ & & & No & NO & No & NO \\
\hline & 13 & $\begin{array}{l}\text { Conexion del MP } \\
\text { a la linea de } \\
\text { administracion }\end{array}$ & & & si & No & SI (derrame y exposición del profesional si no clampado o purgado adecuadamente) & No \\
\hline & 14 & $\begin{array}{l}\text { Indicar velocidad infusión en bomba } \\
\text { (si administración con bomba) (volumen/tiempo) }\end{array}$ & & & No & No & No & No \\
\hline & 15 & $\begin{array}{l}\text { Desclampaie MP } \\
\text { (solos si equipo de infusión tipo árbol) }\end{array}$ & & & No & No & No & No \\
\hline & \multirow[t]{5}{*}{$\# 16$} & $\begin{array}{l}\text { 16.1. Administrtación IV (sistemas de administración tipo árbol. Equipo } \\
\text { secundario (alargadera) conectado a la bolsa de diluyente del MP } \\
\text { mediante punzzon) }\end{array}$ & & & si & No & SI (fuga a través del punzón de conexión de la linea secundaria al MP). & NO \\
\hline & & 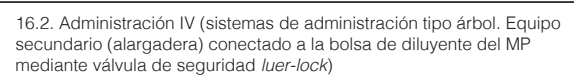 & & & No & No & No & NO \\
\hline & & $\begin{array}{l}\text { 16.3. Administración IV } \\
\text { (sistemas de administración valvulares. Conexión al diluyente del MP } \\
\text { mediante punzón) }\end{array}$ & & & si & No & SI (fuga a través del punzón de conexión al MP) & No \\
\hline & & $\begin{array}{l}\text { 16.4. Administración IV (linea principal de administración con filtro } \\
\text { especial en la cámara de goteo (Air Stop): no permite la entrada de } \\
\text { aire en el tubo de infusión si se vacia la cámara) }\end{array}$ & & & No & No & No & No \\
\hline & & $\begin{array}{l}\text { 16.5. Admininistración nv (linea principal de administración con cámara } \\
\text { de goteo convencional, sin filtro Air Stop) }\end{array}$ & & & si & NO & $\begin{array}{l}\text { Si (entrada de aire en la linea principal de administración si se vacia la cámara de goteo, que hace } \\
\text { necesario el purgadod de la linea a traveses de la punta de conexión al cateteter del paciente, con posible } \\
\text { goteo/derrame del IP) }\end{array}$ & NO \\
\hline & 17 & $\begin{array}{l}\text { Clampaje del MP } \\
\text { (sololo si equipo de infusión tipo árbol) }\end{array}$ & & & No & No & No & No \\
\hline & 18 & $\begin{array}{l}\text { Desclampaje de la SLy } \\
\text { lavadd de linea principal de administración (sólo si equipo de infusión } \\
\text { tipo arbol) }\end{array}$ & & & No & No & No & No \\
\hline & \multirow[t]{3}{*}{$\# 19$} & $\begin{array}{l}\text { 19.1. Desconexión del equipo de administración del paciente (si } \\
\text { sistema de administración tipo árbol y administración por bomba) }\end{array}$ & & & $\mathrm{si}$ & NO & 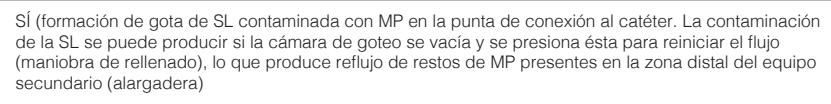 & NO \\
\hline & & $\begin{array}{l}\text { 19.2. Desconexión del equipo de administración del paciente (si } \\
\text { sistema de adminisitración tipo àrbol y administracioion por gravedad) }\end{array}$ & & & si & No & 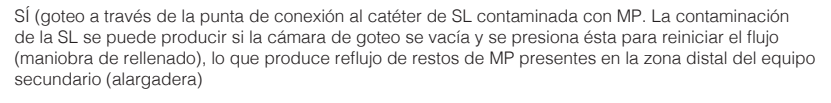 & NO \\
\hline & & $\begin{array}{l}\text { 19.3. Desconexión del MP del equipo de infusión (si sistema de } \\
\text { administración valular) }\end{array}$ & & & $\mathrm{si}$ & NO & $\begin{array}{l}\text { SI (contaminación de la valuula de seguridad que conecta el MP con el sistema de infusión (si } \\
\text { conexiones no secas). }\end{array}$ & No \\
\hline & . & Ir al final de tabla: etapas comunes finales $(20,21$ y 22) & & & - & - & - & - \\
\hline \multirow{6}{*}{ 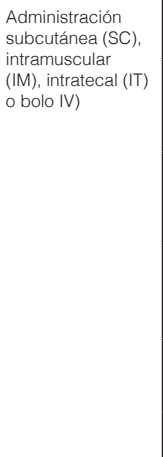 } & . & Ir al inicio de tabla: etapas comunes iniciales $(5,6,7,8$ y 9$)$ & & & - & - & - & - \\
\hline & 23 & $\begin{array}{l}\text { Conexión de la aguja de } \\
\text { administración al conector luer-macho del SCTF (sólo si se utilizan } \\
\text { SCTF tipo macho-hembra) }\end{array}$ & & & No & NO & NO & No \\
\hline & \multirow[t]{2}{*}{$\# 24$} & $\begin{array}{l}\text { 24.1. Conexión del } \\
\text { sistema "aguja- luer macho" al sistema "luer hembra - jeringa" (si se } \\
\text { utilizan SCTFF tipo /uer macho- hembra) }\end{array}$ & & & no & NO & No & NO \\
\hline & & $\begin{array}{l}\text { 24.2. Conexión de la } \\
\text { agujad de administración al sistema "conector / luer-jeringa" con MP (si } \\
\text { se utilizan conectores cerrados luer-lock) }\end{array}$ & & & $\mathrm{si}$ & NO & $\begin{array}{l}\text { SI (sic conexión inadecuada o presión del émbolo antes de conectar la aguja, generándose una } \\
\text { sobrepresión y consecuente derrame al conectar la aguja) }\end{array}$ & NO \\
\hline & 25 & Administración subcutánea, intramuscular, intratecal, bolo intravenoso & & & $\mathrm{si}$ & 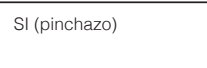 & SI (exposición si derrame) & $\begin{array}{l}\text { SI (pinchazo post- } \\
\text { administración) }\end{array}$ \\
\hline & & Ir al final de tabla: etapas comunes finales $(20,21$ y 22) & & & & & & \\
\hline \multirow[t]{5}{*}{$\begin{array}{l}\text { Administración } \\
\text { intravesical }\end{array}$} & . & Ir a linicio de tabla: etapas comunes iniciales $(5,6,7,8$ y 9$)$ & & & - & - & - & - \\
\hline & 26 & $\begin{array}{l}\text { Conexión a la } \\
\text { sondade de } \\
\text { adminitraín mediante dispositivo cerrado (el dispositivo "cerrado" } \\
\text { vienen ya colocado a la jeringa desde farmacia) }\end{array}$ & & & No & NO & no & NO \\
\hline & 27 & Administración intravesical & & & no & NO & No & No \\
\hline & 28 & Retirada del equipo de administración (sonda + jeringa) & & & $\mathrm{si}$ & NO & SI (goteo de MP a través de la punta de la sonda) & No \\
\hline & * & $\begin{array}{l}\text { Iral final de tabla: etapas comunes finales } \\
(20,21 \text { y } 22)\end{array}$ & & & - & & - & - \\
\hline \multirow{3}{*}{$\begin{array}{l}\text { Administración } \\
\text { oftálmica }\end{array}$} & + & Ir a linicio de tabla: etapas comunes iniciales $(5,6,7,8$ y 9$)$ & & & - & - & - & - \\
\hline & 29 & Administración offálmica & & & $\mathrm{si}$ & NO & SI (exposición del profesional si derrame) & No \\
\hline & * & Ir al final de tabla: etapas comunes finales $(20,21$ y 22) & & & - & - & - & - \\
\hline \multirow{4}{*}{$\begin{array}{l}\text { Administración } \\
\text { oral y tópica }\end{array}$} & * & If a linicio de tabla: etapas comunes iniciciales $(5,6,7,8$ y 9$)$ & & & - & - & - & - \\
\hline & 30 & Administración formas solidas orales & & & no & NO & NO & NO \\
\hline & 31 & Administración formas liquidas orales y formas farmacéuticas tópicas & & & si & NO & SI (exposición si derrame o salpicadura) & No \\
\hline & * & ir a l final de tabla: etapas comunes finales $(20,21$ y 22$)$ & & & - & - & - & - \\
\hline \multirow{3}{*}{ 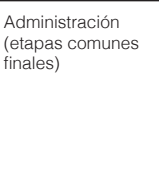 } & 20 & Retirada de EP (4) (5) ó (6) (ver flujograma, figura 1) & & & si & No & SI (exposición del trabajador al MP por retirada incorrecta de EPI) & No \\
\hline & 21 & Gestión de residuos II & & & sı & SI (cortes, pinchazos) & SI (exposición del trabajador a los restos del MP) & $\begin{array}{l}\text { SI (pinchazo } \\
\text { con augij post- } \\
\text { administración) }\end{array}$ \\
\hline & 22 & Lavado de manos II & & & nO & NO & NO & NO \\
\hline
\end{tabular}


Tabla 2. Descripción de riesgos químicos y vías de exposición

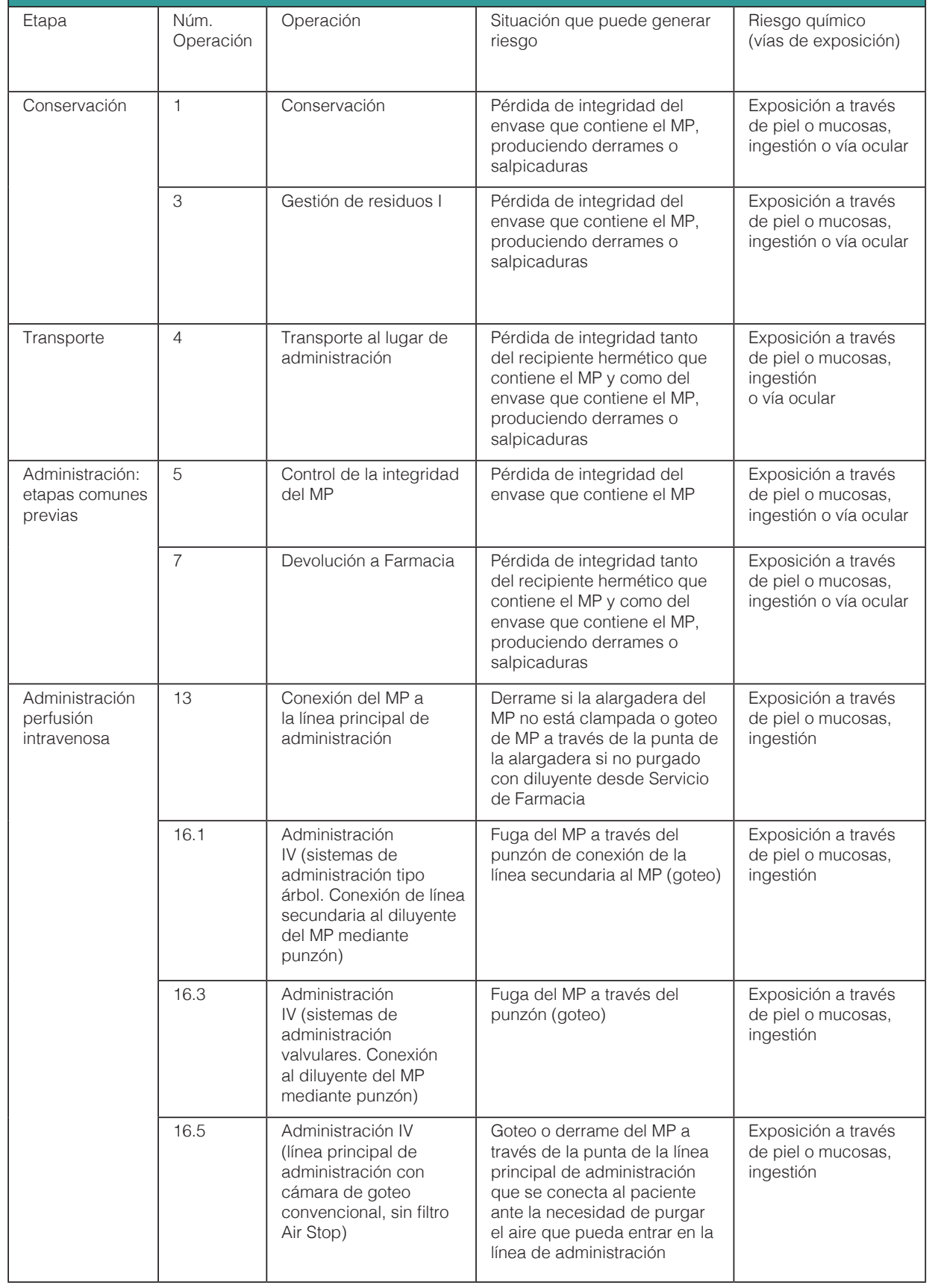




\begin{tabular}{|c|c|c|c|c|}
\hline \multirow[t]{3}{*}{$\begin{array}{l}\text { Administración } \\
\text { perfusión IV }\end{array}$} & 19.1 & $\begin{array}{l}\text { Desconexión del equipo } \\
\text { de administración del } \\
\text { paciente (si sistema } \\
\text { de administración tipo } \\
\text { árbol y administración } \\
\text { por bomba) }\end{array}$ & $\begin{array}{l}\text { Contaminación con MP } \\
\text { de la punta de la línea } \\
\text { principal de administración } \\
\text { al desconectarla del catéter } \\
\text { del paciente, debido a la } \\
\text { contaminación química de la } \\
\text { solución de lavado por reflujo } \\
\text { de restos del MP presentes } \\
\text { en el equipo secundario } \\
\text { (alargadera), al presionar la } \\
\text { cámara de goteo si esta se } \\
\text { queda vacía (maniobra de } \\
\text { rellenado). }\end{array}$ & $\begin{array}{l}\text { Exposición a través } \\
\text { de piel o mucosas, } \\
\text { ingestión }\end{array}$ \\
\hline & 19.2 & $\begin{array}{l}\text { Desconexión del equipo } \\
\text { de administración del } \\
\text { paciente (si sistema } \\
\text { de administración tipo } \\
\text { árbol y administración } \\
\text { por gravedad) }\end{array}$ & $\begin{array}{l}\text { Goteo de MP a través de la } \\
\text { punta de la línea principal } \\
\text { de administración al } \\
\text { desconectarla del catéter } \\
\text { del paciente, debido a la } \\
\text { contaminación química de la } \\
\text { solución de lavado por reflujo } \\
\text { de restos del MP presentes } \\
\text { en el equipo secundario } \\
\text { (alargadera), al presionar la } \\
\text { cámara de goteo si esta se } \\
\text { queda vacía (maniobra de } \\
\text { rellenado) }\end{array}$ & $\begin{array}{l}\text { Exposición a través } \\
\text { de piel o mucosas, } \\
\text { ingestión }\end{array}$ \\
\hline & 19.3 & $\begin{array}{l}\text { Desconexión del } \\
\text { MP del equipo de } \\
\text { infusión (si sistema de } \\
\text { administración valvular) }\end{array}$ & $\begin{array}{l}\text { Contaminación de la válvula } \\
\text { de seguridad que conecta } \\
\text { el MP con el sistema de } \\
\text { infusión (si conexiones no } \\
\text { secas). La contaminación } \\
\text { de la SL se puede producir } \\
\text { si la cámara de goteo se } \\
\text { vacía y se presiona ésta } \\
\text { para reiniciar el flujo, lo que } \\
\text { produce aspiración de restos } \\
\text { de MP presentes en la zona } \\
\text { distal del equipo secundario } \\
\text { (alargadera) }\end{array}$ & $\begin{array}{l}\text { Exposición a través } \\
\text { de piel o mucosas, } \\
\text { ingestión }\end{array}$ \\
\hline \multirow[t]{2}{*}{$\begin{array}{l}\text { Administración- } \\
\text { etapas comunes } \\
\text { final }\end{array}$} & 20 & Retirada de EPI & $\begin{array}{l}\text { Contacto con restos del } \\
\text { MP que hayan quedado } \\
\text { contaminado los EPI }\end{array}$ & $\begin{array}{l}\text { Exposición a través } \\
\text { de piel o mucosas, } \\
\text { ingestión }\end{array}$ \\
\hline & 21 & Gestión de residuos II & $\begin{array}{l}\text { Pinchazo al eliminar en } \\
\text { contenedores jeringas con } \\
\text { aguja }\end{array}$ & Parenteral \\
\hline \multirow[t]{2}{*}{$\begin{array}{l}\text { Administración } \\
\text { SC, IM, bolo IV } \\
\text { e IT }\end{array}$} & 24.2 & $\begin{array}{l}\text { Conexión de la aguja } \\
\text { de administración al } \\
\text { sistema "conector } \\
\text { luer-jeringa" con MP (si } \\
\text { se utilizan conectores } \\
\text { cerrados luer-lock) }\end{array}$ & $\begin{array}{l}\text { Derrame del MP si colocación } \\
\text { inadecuada de la aguja } \\
\text { o si por error se presiona } \\
\text { el émbolo a la vez que se } \\
\text { conecta la aguja }\end{array}$ & $\begin{array}{l}\text { Exposición a través } \\
\text { de piel o mucosas, } \\
\text { ingestión }\end{array}$ \\
\hline & 25 & $\begin{array}{l}\text { Administración SC, IM, } \\
\text { bolo IV e IT }\end{array}$ & $\begin{array}{l}\text { Derrame del MP, salpicadura } \\
\text { y pinchazo }\end{array}$ & $\begin{array}{l}\text { Exposición a través } \\
\text { de piel o mucosas, } \\
\text { ingestión, vía ocular y } \\
\text { parenteral }\end{array}$ \\
\hline
\end{tabular}




\begin{tabular}{|l|l|l|l|l|}
\hline $\begin{array}{l}\text { Administración } \\
\text { intravesical }\end{array}$ & 28 & $\begin{array}{l}\text { Retirada del equipo } \\
\text { de administración } \\
\text { completo, en bloque } \\
\text { (sonda vesical + } \\
\text { jeringa) }\end{array}$ & $\begin{array}{l}\text { Goteo del MP a través de la } \\
\text { punta de la sonda }\end{array}$ & $\begin{array}{l}\text { Exposición a través } \\
\text { de piel o mucosas, } \\
\text { ingestión }\end{array}$ \\
\hline $\begin{array}{l}\text { Administración } \\
\text { oftálmica }\end{array}$ & 29 & $\begin{array}{l}\text { Administración } \\
\text { oftálmica }\end{array}$ & $\begin{array}{l}\text { Derrame del MP contenido en } \\
\text { el envase de colirio o jeringa } \\
\text { intravítrea }\end{array}$ & $\begin{array}{l}\text { Exposición a través } \\
\text { de piel o mucosas, } \\
\text { ingestión }\end{array}$ \\
\hline $\begin{array}{l}\text { Administración } \\
\text { oral y tópica }\end{array}$ & 31 & $\begin{array}{l}\text { Administración formas } \\
\text { líquidas orales y formas } \\
\text { farmacéuticas tópicas }\end{array}$ & $\begin{array}{l}\text { Exposición si derrame o } \\
\text { salpicadura }\end{array}$ & $\begin{array}{l}\text { Exposición a través } \\
\text { de piel o mucosas, } \\
\text { ingestión o vía ocular. }\end{array}$ \\
\hline
\end{tabular}

